\title{
The impact of two doses of coenzyme Q10 on semen parameters and antioxidant status in men with idiopathic oligoasthenoteratozoospermia
}

\author{
Ahmed T Alahmar \\ Department of Pathological Analysis, College of Science, University of Sumer, Rifai, Iraq
}

Objective: Oxidative stress contributes to male infertility, and antioxidants have been recommended for treating idiopathic oligoasthenoteratozoospermia (OAT). There is, however, a lack of agreement on the type, dosing, and use of individual antioxidants or combinations thereof. The purpose of this study was to compare the effects of two doses of coenzyme Q10 (CoQ10) on semen parameters and antioxidant status in men with idiopathic OAT.

Methods: In this prospective study, patients with idiopathic OAT received $200 \mathrm{mg} /$ day $(\mathrm{n}=35)$ or $400 \mathrm{mg} /$ day $(\mathrm{n}=30)$ of CoQ10 orally for 3 months. All patients underwent semen analysis according to the fifth editions of the World Health Organization criteria. Total antioxidant capacity (TAC), catalase (CAT) activity, and superoxide dismutase (SOD) activity were measured both before and after treatment.

Results: Treatment with CoQ10 (200 mg/day or $400 \mathrm{mg} /$ day) resulted in a significant increase in sperm concentration from baseline ( $8.22 \pm 6.88$ to $12.53 \pm 8.11 \mathrm{million} / \mathrm{mL}, p=0.019 ; 7.58 \pm 5.41$ to $12.33 \pm 6.1 \mathrm{million} / \mathrm{mL}, p=0.002$, respectively), progressive motility $(16.54 \% \pm 9.26 \%$ to $22.58 \% \pm 10.15 \%, p=0.011 ; 14.22 \% \pm 12.85 \%$ to $26.1 \% \pm 14.52 \%, p=0.001$, respectively), and total motility ( $25.68 \% \pm 6.41 \%$ to $29.96 \% \pm 8.09 \%, p=0.016 ; 23.46 \% \pm 12.59 \%$ to $34.82 \% \pm 14.17 \%, p=0.001$, respectively). CoQ10 therapy also increased $\operatorname{TAC}(p=0.009, p=0.001$, respectively), SOD activity ( $p=0.004, p=0.001$, respectively), and CAT activity $(p=0.039, p=0.024$, respectively). Furthermore, antioxidant measures correlated significantly with seminal fluid parameters $(r=0.36-0.76)$.

Conclusion: CoQ10 supplementation improved semen parameters and antioxidant status in men with idiopathic OAT, with a greater improvement shown in men who took $400 \mathrm{mg} /$ day than in those who took $200 \mathrm{mg} /$ day.

Keywords: Antioxidants; Coenzyme Q10; Oxidative stress; Semen

\section{Introduction}

The prevalence of infertility has increased significantly in recent decades, and it is currently estimated to affect approximately $15 \%$ of the population worldwide [1]. Male infertility has been linked to many factors, including varicocele, infections, undescended testis,

Received: Mar 22, 2019· Revised: May 8, 2019· Accepted: May 9, 2019 Corresponding author: Ahmed T Alahmar

Department of Pathological Analysis, College of Science, University of Sumer, Rifai 64005 , Iraq

Tel:+964-7808180900 E-mail:ahmed.t.alahmar@gmail.com

This is an Open Access article distributed under the terms of the Creative Commons Attribution Non-Commercial License (http://creativecommons.org/licenses/by-nc/4.0/) which permits unrestricted non-commercial use, distribution, and reproduction in any medium, provided the original work is properly cited. and autoimmune, endocrine, genetic, and environmental factors [2]. However, no underlying cause for infertility is identified in 30\%-40\% of patients; such cases are termed idiopathic male infertility (IMI). Potential mechanisms underlying IMI include genetic, biochemical, hormonal, and environmental factors [3].

Oxidative stress (OS) has been proposed as a mechanism underlying IMI. OS is defined as a disruption of the prooxidant-antioxidant balance that leads to DNA damage, peroxidation of plasma membrane lipids, and protein oxidation [4]. OS occurs when reactive oxygen species (ROS) disrupt the equilibrium of reduction and oxidation. ROS are highly reactive molecules produced during normal cellular metabolism and physiology, formed due to the incomplete reduction of oxygen [5]. In seminal plasma, ROS play a fundamental physi- 
ologic role in several sperm functions, such as the development, maturation, and capacitation of spermatozoa, as well as the acrosome reaction and fertilization [6]. Sperm cells and leukocytes are the main sources of ROS in seminal plasma. Although adequate amounts of ROS are crucial for normal sperm function, excess levels trigger OS and negatively affect reproductive outcomes [4,7]. ROS affect sperm motility by altering axonemal protein phosphorylation and sperm membrane fluidity. Furthermore, ROS may induce sperm DNA damage, including DNA deletion, cross-linking, and chromosome rearrangement, leading to impaired fertilization, abnormal embryonic development, and possibly, congenital foetal defects.

Spermatozoa have a limited intrinsic antioxidant capacity and DNA repair system [8]. However, semen contains several endogenous and exogenous antioxidant molecules that allow maintenance of the balance between reduction and oxidation, including catalase (CAT), superoxide dismutase (SOD), glutathione peroxidase, vitamin C, vitamin E, coenzyme Q10 (CoQ10), carnitine, selenium, zinc, copper, and carotenoids $[9,10]$. Nevertheless, in men with IMI, poor semen quality is associated with decreased antioxidant capacity [11]. Both increased ROS production and reduced seminal plasma antioxidant capacity have been reported in infertile men [12].

CoQ10 is a lipid-soluble compound synthesized de novo, which may be found in a reduced (ubiquinol) or oxidized (ubiquinone) form [1]. Endogenous CoQ10 levels are closely related to overall oxidative activity, as it plays a role in mitochondrial bioenergetics as part of the mitochondrial respiratory chain [13]. CoQ10 also modulates gene expression, cell signalling, transport, and metabolism. Moreover, the reduced form acts as an electron acceptor in plasma and subcellular fractions [14].

Studies have demonstrated low levels of CoQ10 in infertile men $[1,3]$. Further, CoQ10 supplementation was associated with improvement of 1 or more seminal fluid parameters [15]. A placebo-controlled study found that in infertile men with oligoasthenoteratozoospermia (OAT), CoQ10 treatment improved semen parameters and antioxidant status [16]. A meta-analysis by Lafuente et al. [3] reported improvements in sperm concentration and motility, as well as an increase in seminal CoQ10 levels. Several studies have shown beneficial effects of antioxidant treatment in infertile men [17-19]. However, there is no consensus on the type, dosing, patient selection, and the use of individual or combination therapy [20]. In this context, the objective of this study was to evaluate the effect of oral CoQ10 at doses of $200 \mathrm{mg} /$ day or $400 \mathrm{mg} /$ day for 3 months on sperm parameters and seminal plasma antioxidant status in infertile men with idiopathic OAT.

\section{Methods}

\section{Patients}

Out of 70 patients initially selected for the study, 65 patients (mean age, $27.24 \pm 7.81$ years) with idiopathic OAT were recruited at the Fertility Clinic, Babil Governorate, Iraq from June to November 2018 and enrolled in the study (five patients did not complete the study). All patients underwent a medical assessment including a history, physical examination, and laboratory and radiological investigations. The study was conducted as a prospective randomized clinical trial with 3 months of follow-up. The selected patients who fulfilled the selection criteria were randomly assigned to two treatment groups. Group 1 ( $n=35$ ) received $200 \mathrm{mg}$ (single dose) of oral CoQ10 and group 2 ( $n=30$ ) received $400 \mathrm{mg}$ (single dose) of oral CoQ10 (in the reduced form ubiquinol; America Medic and Science, Woodinville, WA, USA) daily for 3 months. The first group served as active control. We chose CoQ10 doses analysed in previous studies [1,21]. Semen analysis was performed, and total antioxidant capacity (TAC), CAT, and SOD activity were measured in seminal plasma samples and compared before and after therapy.

\section{Eligibility criteria}

The inclusion criteria comprised a history of infertility lasting for at least 12 months despite regular unprotected intercourse. OAT was diagnosed by semen analysis results showing abnormal sperm concentration $(<15$ million $/ \mathrm{mL}$ ), progressive motility $(<32 \%)$, and total motility $(<40 \%)$ as defined by the fifth edition of the World Health Organization (WHO) criteria for semen analysis [22] and abnormal morphology ( $<30 \%$ normal morphology) as defined by the fourth edition of the WHO criteria [23]. The exclusion criteria comprised azoospermia, varicocele, genital tract infection, cryptorchidism, testicular trauma or scrotal surgery, endocrine disorders, systemic illness including hepatic and renal diseases, smoking, recent intake of antioxidants, and the presence of female factor infertility.

\section{Semen analysis}

Semen samples were obtained by masturbation after sexual abstinence for 2-3 days, collected in a sterile wide-mouth plastic container, kept at $37^{\circ} \mathrm{C}$ until liquefaction, and then analysed within 1 hour of production using the manual method according to fifth edition of the WHO criteria [22] and the fourth edition criteria [23] for sperm morphology. Semen volume, sperm concentration, motility, and morphology were measured. All semen analyses were performed by the same investigator for the sake of data consistency, and all patients underwent two semen analyses before and after therapy; the average values were used. 


\section{Seminal TAC}

Semen samples were centrifuged at 3,000 rpm for 5 minutes, and the seminal plasma was aspirated and stored frozen for further biochemical analysis. TAC was measured by a colorimetric method using the total antioxidant capacity assay kit (\#E-BC-K136; Elabscience, Houston, TX, USA). The test is based on the principle that antioxidants in the body can reduce $\mathrm{Fe}^{3+}$ to $\mathrm{Fe}^{2+}$ and that $\mathrm{Fe}^{2+}$ can form stable complexes with phenanthroline. The TAC was calculated by measuring the absorbance at $520 \mathrm{~nm}$ using a standard protocol.

\section{Seminal SOD activity}

SOD activity was determined by a colorimetric method as described by Magnani et al. [24]. The principle of this method is based on competition between pyrogallol autoxidation by superoxide anion and scavenging of this radical by SOD. The activity of SOD was calculated by measuring the absorbance at $420 \mathrm{~nm}$ using a standard protocol.

\section{Seminal CAT activity}

Seminal CAT activity was determined by a colorimetric method using the CAT assay kit (\#E-BC-K031, Elabscience). The test is based on the principle that the process through which CAT decomposes $\mathrm{H}_{2} \mathrm{O}_{2}$ can be quickly stopped by ammonium molybdate. The residual $\mathrm{H}_{2} \mathrm{O}_{2}$ reacts with ammonium molybdate to generate a yellowish complex. CAT activity was calculated by measuring the absorbance of the yellowish complex at $405 \mathrm{~nm}$ using a standard protocol.

\section{Statistical analysis}

IBM SPSS ver. 24.0 (IBM Corp., Armonk, NY, USA) was used for all statistical analyses. The results were expressed as mean \pm standard deviation. The Kolmogorov-Smirnov test was used to assess the normality of the data. The paired $t$-test was used to compare mean values before and after treatment. The Pearson correlation coefficient $(r)$ was used to evaluate correlations between seminal parameters and
TAC, CAT, and SOD. The $p$-values less than 0.05 were considered to indicate statistical significance.

\section{Human and animal rights}

All procedures followed were in accordance with the ethical standards of the responsible committee on human experimentation (institutional and national) and with the Helsinki Declaration of 1975, as revised in 2008. The study protocol was approved by local Ethical Committee at University of Sumer, Iraq and all participants provided informed consent for participation in the study NCT03850561 (https:// clinicaltrials.gov/).

\section{Results}

A total of 65 patients were included in the study, with 35 patients in group 1 and 30 patients in group 2. The mean age was $26.23 \pm 7.22$ and $29.45 \pm 8.61$ years in groups 1 and 2 , respectively $(p=0.094)$, while the mean duration of infertility was $4.34 \pm 3.64$ and $4.91 \pm 4.17$ years in groups 1 and 2, respectively ( $p=0.54$ ) (Table 1). Treatment with CoQ10 (200 mg or $400 \mathrm{mg}$ per day) resulted in a significant increase in sperm concentration from baseline $(8.22 \pm 6.88$ to $12.53 \pm 8.11$ million $/ \mathrm{mL}$, $p=0.019 ; 7.58 \pm 5.41$ to $12.33 \pm 6.1 \mathrm{million} / \mathrm{mL}, p=0.002$, respectively), as well as improvements in progressive motility $(16.54 \% \pm 9.26 \%$ to $22.58 \% \pm 10.15 \%, p=0.011 ; 14.22 \% \pm 12.85 \%$ to $26.1 \% \pm 14.52 \%$, $p=0.001$, respectively), and total motility $(25.68 \% \pm 6.41 \%$ to $29.96 \%$ $\pm 8.09 \%, p=0.016 ; 23.46 \% \pm 12.59 \%$ to $34.82 \% \pm 14.17 \%, p=0.001$ respectively). These changes were greater in the group treated with $400 \mathrm{mg}$ of CoQ10 (Table 1).

Likewise, treatment with CoQ10 (200 mg/day or $400 \mathrm{mg} /$ day) resulted in significant increases in TAC $(p=0.009, p=0.001$, respectively), SOD activity ( $p=0.004, p=0.001$, respectively), and CAT activity $(p=0.039, p=0.024$, respectively), with greater changes in subjects treated with $400 \mathrm{mg}$ of CoQ10 (Table 2).

Furthermore, in group 1 (200 mg/day), significant positive correla-

Table 1. Patient characteristics and semen parameters before and after CoQ10 treatment ( 200 and $400 \mathrm{mg} / \mathrm{day}$ )

\begin{tabular}{|c|c|c|c|c|c|c|}
\hline \multirow{2}{*}{ Variable } & \multicolumn{2}{|c|}{ CoQ10 $200 \mathrm{mg} /$ day $(\mathrm{n}=35)$} & \multirow{2}{*}{$p$-value } & \multicolumn{2}{|c|}{ CoQ10 400 mg/day $(n=30)$} & \multirow{2}{*}{$p$-value } \\
\hline & Before & After & & Before & After & \\
\hline Age (yr) & $26.23 \pm 7.22$ & & & $29.45 \pm 8.61$ & & 0.094 \\
\hline Duration of infertility (yr) & $4.34 \pm 3.64$ & & & $4.91 \pm 4.17$ & & 0.54 \\
\hline Volume (mL) & $2.38 \pm 1.30$ & $2.51 \pm 1.42$ & 0.69 & $2.15 \pm 1.68$ & $2.28 \pm 1.35$ & 0.74 \\
\hline Concentration (million $/ \mathrm{mL}$ ) & $8.22 \pm 6.88$ & $12.53 \pm 8.11$ & 0.019 & $7.58 \pm 5.41$ & $12.33 \pm 6.1$ & 0.002 \\
\hline Progressive motility (\%) & $16.54 \pm 9.26$ & $22.58 \pm 10.15$ & 0.011 & $14.22 \pm 12.85$ & $26.1 \pm 14.52$ & 0.001 \\
\hline Total motility (\%) & $25.68 \pm 6.41$ & $29.96 \pm 8.09$ & 0.016 & $23.46 \pm 12.59$ & $34.82 \pm 14.17$ & 0.001 \\
\hline Normal morphology (\%) & $22.17 \pm 6.08$ & $23.64 \pm 7.45$ & 0.36 & $24.64 \pm 4.93$ & $27.41 \pm 4.58$ & 0.27 \\
\hline
\end{tabular}

Values are presented as mean \pm standard deviation.

CoQ10, coenzyme Q10. 
Table 2.TAC, SOD, and CAT activity in seminal plasma before and after CoQ10 treatment

\begin{tabular}{|c|c|c|c|c|c|c|}
\hline \multirow{2}{*}{ Variable } & \multicolumn{2}{|c|}{ CoQ10 200 mg/day $(n=35)$} & \multirow{2}{*}{$p$-value } & \multicolumn{2}{|c|}{ CoQ10 400 mg/day $(n=30)$} & \multirow{2}{*}{$p$-value } \\
\hline & Before & After & & Before & After & \\
\hline $\mathrm{TAC}(\mathrm{mmol} / \mathrm{L})$ & $1.1 \pm 0.30$ & $1.28 \pm 0.26$ & 0.009 & $0.92 \pm 0.6$ & $1.58 \pm 0.83$ & 0.001 \\
\hline $\mathrm{SOD}(\mathrm{U} / \mathrm{mL})$ & $12.6 \pm 3.71$ & $15.4 \pm 4.31$ & 0.004 & $10.58 \pm 4.29$ & $14.26 \pm 4.29$ & 0.001 \\
\hline CAT $(\mathrm{U} / \mathrm{mL})$ & $11.3 \pm 2.53$ & $12.5 \pm 2.24$ & 0.039 & $11.8 \pm 3.17$ & $13.6 \pm 2.88$ & 0.024 \\
\hline
\end{tabular}

Values are presented as mean \pm standard deviation.

TAC, total antioxidant capacity; SOD, superoxide dismutase; CAT, catalase; CoQ10, coenzyme Q10.

Table 3. Correlations between semen parameters and antioxidant parameters in patients in group 1

\begin{tabular}{|c|c|c|c|c|c|c|}
\hline \multirow{2}{*}{ Variable } & \multicolumn{2}{|c|}{ Concentration } & \multicolumn{2}{|c|}{ Motility } & \multicolumn{2}{|c|}{ Morphology } \\
\hline & $r^{a)}$ & $p$-value & $r^{\mathrm{a})}$ & $p$-value & $r^{\mathrm{a})}$ & $p$-value \\
\hline TAC & 0.52 & 0.008 & 0.76 & 0.001 & 0.37 & 0.04 \\
\hline SOD & 0.46 & 0.022 & 0.54 & 0.006 & 0.4 & 0.034 \\
\hline CAT & 0.41 & 0.028 & 0.48 & 0.014 & 0.34 & 0.08 \\
\hline
\end{tabular}

TAC, total antioxidant capacity; SOD, superoxide dismutase; CAT, catalase.

a)Pearson correlation coefficient.

Table 4. Correlations between semen parameters and antioxidant parameters in patients in group 2

\begin{tabular}{|c|c|c|c|c|c|c|}
\hline \multirow{2}{*}{ Variable } & \multicolumn{2}{|c|}{ Concentration } & \multicolumn{2}{|c|}{ Motility } & \multicolumn{2}{|c|}{ Morphology } \\
\hline & $r^{2)}$ & $p$-value & $r^{a)}$ & $p$-value & $r^{\text {r) }}$ & $p$-value \\
\hline TAC & 0.55 & 0.005 & 0.68 & 0.002 & 0.31 & 0.07 \\
\hline SOD & 0.49 & 0.009 & 0.61 & 0.003 & 0.29 & 0.09 \\
\hline CAT & 0.38 & 0.02 & 0.53 & 0.005 & 0.36 & 0.04 \\
\hline
\end{tabular}

TAC, total antioxidant capacity; SOD, superoxide dismutase; CAT, catalase.

a)Pearson correlation coefficient.

tions were found between sperm concentration and TAC $(r=0.52$, $p=0.008)$, SOD activity ( $r=0.46, p=0.022)$, and CAT activity ( $r=0.41$, $p=0.028$ ). Similar correlations were between sperm motility and TAC $(r=0.76, p=0.001)$, SOD activity $(r=0.54, p=0.006)$ and CAT activity $(r=0.48, p=0.014)$, as well as between normal sperm morphology and TAC ( $r=0.37, p=0.04)$ and SOD activity $(r=0.4, p=0.034)$ (Table 3). The strongest correlations were found between sperm concentration, motility, and antioxidant measures.

Moreover, in group 2 (400 mg/day), significant positive correlations were found between sperm concentration and TAC $(r=0.55$, $p=0.005)$, SOD activity $(r=0.49, p=0.009)$, and CAT activity ( $r=0.38$, $p=0.02)$; between sperm motility and TAC ( $r=0.68, p=0.002)$, SOD activity ( $r=0.61, p=0.003)$, and CAT activity $(r=0.53, p=0.005)$; as well as between normal sperm morphology and CAT activity $(r=0.36$, $p=0.04$ ) (Table 4). The strongest correlations were likewise found between sperm concentration, motility, and antioxidant measures.

\section{Discussion}

The present study demonstrated that the reduced form of CoQ10 exerted beneficial effects on sperm concentration, motility, and antioxidant capacity in men with idiopathic OAT. Greater changes were detected in response to a dose of $400 \mathrm{mg} /$ day. To our knowledge, this is the first study to compare the effect of two doses of CoQ10 on seminal plasma antioxidant capacity.

Our findings are consistent with previous reports. Exogenous intake of CoQ10 may increase its levels in seminal plasma and enhance sperm function [1]. Treatment with ubiquinol (100 mg twice a day) for 6 months improved sperm motility and morphology in patients with idiopathic OAT [25]. Further, in a randomized double-blind placebo-controlled clinical trial in men with men with idiopathic asthenospermia who received CoQ10 (200 mg daily) for 6 months, significant improvements were observed in all seminal parameters, along with increased CoQ10 levels in seminal plasma [1]. Similar findings were reported by Safarinejad and colleagues $[16,26]$ after administration of CoQ10 (200 mg) for 26 weeks in men with OAT and $300 \mathrm{mg}$ daily for 26 weeks in men with OAT. Balercia et al. [1,6] reported an increase in CoQ10 levels in seminal plasma and improvements in sperm parameters following administration of CoQ10 (200 mg/day) for 6 months. In contrast, in an open-label prospective study, men 
with OAT who were treated with CoQ10 $(600 \mathrm{mg})$ for 12 months showed significant improvements in sperm progressive motility, concentration, and morphology at the 12-month follow-up [27].

Adequate CoQ10 levels are necessary for proper spermatozoa function given the role of $\mathrm{CoQ} 10$ in the mitochondrial respiratory chain and its antioxidant properties. In particular, mitochondrial dysfunction in spermatozoa has been associated with reduced sperm motility; and OS has been shown to be related with mitochondrial DNA deletions [28]. OS is detrimental for sperm parameters, DNA integrity, and fertilization [20]. Therefore, the improvements in sperm parameters detected in the present study could be attributed to the antioxidant properties of $\mathrm{CoQ10}$, leading to reduced OS and enhanced mitochondrial reduction-oxidation function. Previous studies have also linked dietary supplementation with CoQ10 to increased levels of CoQ10 in circulating lipoproteins and enhanced resistance of human low-density lipoprotein to lipid peroxidation [29]. Increased sperm concentration and motility may enhance fertility potential and pregnancy outcomes. A body of literature has demonstrated beneficial effects of CoQ10 and other antioxidants on semen parameters. However, there is a lack of agreement on the type and the use of individual or combination antioxidant therapy [18,30]. A recent meta-analysis also showed that doses of CoQ10 varied (200-300 mg/day) [15]. In addition, a head-head comparison is not always possible due to the heterogeneity of studies in terms of methodology, patient selection, controls, and the dosing and duration of CoQ10 therapy.

The observed improvement in seminal fluid parameters in the current study was higher with a CoQ10 dose of $400 \mathrm{mg}$ per day. Indeed, CoQ10 plays a central role both as an antioxidant and as a facilitator of adenosine triphosphate synthesis [8]. Thus, it is likely that a greater dose and length of exposure yield stronger effects [21]. This is supported by the meta-analysis of Lafuente et al. [3], in which pooled data from three trials revealed that $\mathrm{CoQ} 10$ treatment significantly enhanced multiple sperm quality parameters. The studies above also used variable doses of $\mathrm{COQ} 10$ for different durations, highlighting the absence of consensus on the optimal dose for $\mathrm{CoQ} 10$ in infertile men. If the optimal effective dose is determined, this treatment is inexpensive, safe, and easy to administer. Furthermore, optimisation of CoQ10 dosing is essential, as recent reports indicated that excessive intake of antioxidants may shift the reduction-oxidation balance to reductive stress, which is as harmful as OS [31]. Once the positive effects of antioxidant supplementation are confirmed, it remains necessary to optimize the dose and duration of treatment and to identify which seminal parameters benefit the most from certain antioxidants. In patients experiencing high levels of $\mathrm{OS}$, doses should be taken for a minimum of 3 months, as the maturation of sperm takes around 72 days. Based on this study, a $400 \mathrm{mg}$ dosage of CoQ10 could be suggested as effective dosage for treatment of patients with idiopathic OAT.
In our study, we also observed enhancements of the oxidative environment, as reflected by the improvement of TAC, SOD and CAT levels, as well as the positive correlations between these variables and semen parameters after 3 months of $\mathrm{COQ} 10$ treatment. Previous studies have demonstrated lower TAC, SOD and CAT levels in infertile men than in fertile men [13,32]. In addition, a meta-analysis by Huang et al. [12] reviewed studies on 3,819 male infertility patients and showed lower levels of antioxidant activity, including TAC, SOD, and CAT, in men with infertility. Previous reports have also detected an increase in antioxidant capacity following antioxidant therapy $[17,33]$. However, studies of the effects of CoQ10 intake on seminal plasma antioxidant levels are limited. Our findings are congruent with those of Nadjarzadeh et al. [13], who showed that CoQ10 administration (200 mg/day for 3 months) increased SOD and CAT activity in the seminal plasma of infertile men. Furthermore, a positive correlation was found between seminal CoQ10 concentrations and semen parameters [26]. In contrast, in a study by Eroglu et al. [34], basal CoQ10 levels in seminal fluid did not appear to be associated with sperm quality parameters or TAC.

The increased TAC, SOD and CAT activities in our study could partially explain the improvement in sperm parameters after treatment with $\mathrm{CoQ10}$. Although $\mathrm{CoQ} 10$ has been recognized as a powerful mediator of cell signalling [35], no specific mechanisms have been identified concerning CoQ10-mediated upregulation of TAC and SOD and CAT activity [36]. Interestingly, CoQ10 has been observed to prevent proinflammatory signalling by insulin, interleukin-17, and STAT3 [37], as well as tumour necrosis factor alpha and various chemokines [38]. In turn, this may reflect an enhanced antioxidant status. Future investigations are needed to elucidate the mechanisms of the CoQ10-mediated enhancement of antioxidant capacity. Our study has certain limitations. We did not investigate pregnancy or live birth rates post-therapy, as those were not the primary outcome measures of the study. Dietary regulation was also not measured in this study. Other limitations include the small sample size and lack of long-term follow-up, so further large-scale long-term clinical studies are warranted.

In conclusion, treatment with CoQ10 improved sperm motility, concentration, and semen antioxidant status in infertile men with idiopathic OAT, with a greater improvement observed in response to a dose of $400 \mathrm{mg} /$ day than a dose of $200 \mathrm{mg} /$ day. Supplementation with CoQ10 may enhance the fertility potential and reproductive outcomes of men with idiopathic infertility.

\section{Conflict of interest}

No potential conflict of interest relevant to this article was reported. 


\section{Acknowledgments}

The author would like to express sincere gratitude to all patients who participated in this study for their valuable help in carrying out this work. The author is also very grateful for Dr. Pallav Sengupta, Mahsa University College, Kuala Lumpur, Malaysia for reviewing this manuscript prior to submission.

\section{ORCID}

Ahmed T Alahmar https://orcid.org/0000-0003-2100-5807

\section{References}

1. Balercia G, Buldreghini E, Vignini A, Tiano L, Paggi F, Amoroso S, et al. Coenzyme Q10 treatment in infertile men with idiopathic asthenozoospermia: a placebo-controlled, double-blind randomized trial. Fertil Steril 2009;91:1785-92.

2. Leaver RB. Male infertility: an overview of causes and treatment options. Br J Nurs 2016;25:S35-40.

3. Lafuente R, Gonzalez-Comadran M, Sola I, Lopez G, Brassesco M, Carreras R, et al. Coenzyme Q10 and male infertility: a metaanalysis. J Assist Reprod Genet 2013;30:1147-56.

4. Majzoub A, Arafa M, Mahdi M, Agarwal A, Al Said S, Al-Emadi I, et al. Oxidation-reduction potential and sperm DNA fragmentation, and their associations with sperm morphological anomalies amongst fertile and infertile men. Arab J Urol 2018;16:87-95.

5. Talevi R, Barbato V, Fiorentino I, Braun S, Longobardi S, Gualtieri R. Protective effects of in vitro treatment with zinc, $d$-aspartate and coenzyme q10 on human sperm motility, lipid peroxidation and DNA fragmentation. Reprod Biol Endocrinol 2013;11:81.

6. Balercia G, Mosca F, Mantero F, Boscaro M, Mancini A, RicciardoLamonica G, et al. Coenzyme Q(10) supplementation in infertile men with idiopathic asthenozoospermia: an open, uncontrolled pilot study. Fertil Steril 2004;81:93-8.

7. Agarwal A, Sharma RK, Sharma R, Assidi M, Abuzenadah AM, Alshahrani $S$, et al. Characterizing semen parameters and their association with reactive oxygen species in infertile men. Reprod Biol Endocrinol 2014;12:33.

8. Mancini A, De Marinis L, Littarru GP, Balercia G. An update of Coenzyme Q10 implications in male infertility: biochemical and therapeutic aspects. Biofactors 2005;25:165-74.

9. Walczak-Jedrzejowska R, Wolski JK, Slowikowska-Hilczer J. The role of oxidative stress and antioxidants in male fertility. Cent European J Urol 2013;66:60-7.

10. Atig F, Raffa M, Habib BA, Kerkeni A, Saad A, Ajina M. Impact of seminal trace element and glutathione levels on semen quality of Tunisian infertile men. BMC Urol 2012;12:6.

11. Samplaski MK, Clemesha CG. Discrepancies between the internet and academic literature regarding vitamin use for male infertility. Transl Androl Urol 2018;7(Suppl 2):S193-7.

12. Huang C, Cao X, Pang D, Li C, Luo Q, Zou Y, et al. Is male infertility associated with increased oxidative stress in seminal plasma? Ameta analysis. Oncotarget 2018;9:24494-513.

13. Nadjarzadeh A, Shidfar F, Amirjannati N, Vafa MR, Motevalian SA, Gohari MR, et al. Effect of Coenzyme Q10 supplementation on antioxidant enzymes activity and oxidative stress of seminal plasma: a double-blind randomised clinical trial. Andrologia 2014;46:177-83.

14. Ahmadi S, Bashiri R, Ghadiri-Anari A, Nadjarzadeh A. Antioxidant supplements and semen parameters: an evidence based review. Int J Reprod Biomed (Yazd) 2016;14:729-36.

15. Salas-Huetos A, Rosique-Esteban N, Becerra-Tomas N, Vizmanos B, Bullo M, Salas-Salvado J. The effect of nutrients and dietary supplements on sperm quality parameters: a systematic review and meta-analysis of randomized clinical trials. Adv Nutr 2018;9:833-48.

16. Safarinejad MR, Safarinejad S, Shafiei N, Safarinejad S. Effects of the reduced form of coenzyme Q10 (ubiquinol) on semen parameters in men with idiopathic infertility: a double-blind, placebo controlled, randomized study. J Urol 2012;188:526-31.

17. Alahmar AT. The effects of oral antioxidants on the semen of men with idiopathic oligoasthenoteratozoospermia. Clin Exp Reprod Med 2018;45:57-66.

18. Majzoub A, Agarwal A. Systematic review of antioxidant types and doses in male infertility: benefits on semen parameters, advanced sperm function, assisted reproduction and live-birth rate. Arab J Urol 2018;16:113-24.

19. Tremellen K. Antioxidant therapy for the enhancement of male reproductive health: a critical review of the literature. In: Parekattil S, Agarwal A, editors. Male infertility. New York: Springer; 2012. p. 389-99.

20. Cardoso JP, Cocuzza M, Elterman D. Optimizing male fertility: oxidative stress and the use of antioxidants. World J Urol 2019;37: 1029-34.

21. Garrido-Maraver J, Cordero MD, Oropesa-Avila M, Fernandez Vega A, de la Mata M, Delgado Pavon A, et al. Coenzyme q10 therapy. Mol Syndromol 2014;5:187-97.

22. World Health Organization. WHO laboratory manual for the examination and processing of human semen. Geneva: World Health Organization; 2010.

23. World Health Organization. WHO laboratory manual for the examination of human semen and sperm-cervical mucus interaction. Cambridge: Cambridge University Press; 1999. 
24. Magnani L, Gaydou EM, Hubaud JC. Spectrophotometric measurement of antioxidant properties of flavones and flavonols against superoxide anion. Anal Chim Acta 2000;411:209-16.

25. Cakiroglu B, Eyyupoglu SE, Gozukucuk R, Uyanik BS. Ubiquinol effect on sperm parameters in subfertile men who have asthenoteratozoospermia with normal sperm concentration. Nephrourol Mon 2014;6:e16870.

26. Safarinejad MR. Efficacy of coenzyme Q10 on semen parameters, sperm function and reproductive hormones in infertile men. J Urol 2009;182:237-48.

27. Safarinejad MR. The effect of coenzyme Q10 supplementation on partner pregnancy rate in infertile men with idiopathic oligoasthenoteratozoospermia: an open-label prospective study. Int Urol Nephrol 2012;44:689-700.

28. Pereira R, Sa R, Barros A, Sousa M. Major regulatory mechanisms involved in sperm motility. Asian J Androl 2017;19:5-14.

29. Mohr D, Bowry VW, Stocker R. Dietary supplementation with coenzyme Q10 results in increased levels of ubiquinol-10 within circulating lipoproteins and increased resistance of human lowdensity lipoprotein to the initiation of lipid peroxidation. Biochim Biophys Acta 1992;1126:247-54.

30. Alahmar AT. Effect of vitamin c, vitamin e, zinc, selenium, and coenzyme Q10 in infertile men with idiopathic oligoasthenozoospermia. Int J Infertil Fetal Med 2017;8:45-9.

31. Henkel R, Sandhu IS, Agarwal A. The excessive use of antioxidant therapy: a possible cause of male infertility? Andrologia 2019; 51:e13162.

32. Pahune PP, Choudhari AR, Muley PA. The total antioxidant power of semen and its correlation with the fertility potential of human male subjects. J Clin Diagn Res 2013;7:991-5.

33. Imamovic Kumalic S, Pinter B. Review of clinical trials on effects of oral antioxidants on basic semen and other parameters in idiopathic oligoasthenoteratozoospermia. Biomed Res Int 2014; 2014:426951.

34. Eroglu M, Sahin S, Durukan B, Ozakpinar OB, Erdinc N, Turkgeldi $\mathrm{L}$, et al. Blood serum and seminal plasma selenium, total antioxidant capacity and coenzyme q10 levels in relation to semen parameters in men with idiopathic infertility. Biol Trace Elem Res 2014;159:46-51.

35. Hernandez-Camacho JD, Bernier M, Lopez-Lluch G, Navas P. Coenzyme $\mathrm{Q}(10)$ supplementation in aging and disease. Front Physiol 2018;9:44.

36. Kang DH, Kang SW. Targeting cellular antioxidant enzymes for treating atherosclerotic vascular disease. Biomol Ther (Seoul) 2013;21:89-96.

37. Lee SY, Lee SH, Yang EJ, Kim JK, Kim EK, Jung K, et al. Coenzyme Q10 inhibits Th17 and STAT3 signaling pathways to ameliorate colitis in mice. J Med Food 2017;20:821-9.

38. Schmelzer C, Lorenz G, Rimbach G, Doring F. In vitro effects of the reduced form of coenzyme $Q(10)$ on secretion levels of TNFalpha and chemokines in response to LPS in the human monocytic cell line THP-1. J Clin Biochem Nutr 2009;44:62-6. 